\title{
Corrigendum to "On generalized open sets"
}

\author{
Serap Erdem ${ }^{1}$ (D), Murad Özkoç ${ }^{* 2}$ (D), Faical Yacine Issaka ${ }^{1}$ (D) \\ ${ }^{1}$ Muğla Sıtkı Koçman University, Graduate School of Natural and Applied Sciences, Mathematics, 48000 \\ Menteşe-Muğla, Turkey \\ ${ }^{2}$ Muğla Sitkı Koçman University, Faculty of Science, Department of Mathematics, 48000 Menteşe-Muğla, \\ Turkey
}

\begin{abstract}
In this corrigendum, we show that Theorem 3.9, Theorem 2.9, Theorem 2.10(1) and Theorem 2.21(9)(10) given in the paper entitled "On generalized open sets" [Hacet. J. Math. Stat. 47(6) (2018), 1438-1446] are incorrect.
\end{abstract}

Mathematics Subject Classification (2020). 54A05, 54A10, 54D99

Keywords. $\gamma$-open set, $\gamma$-semiinterior, $\gamma$-semiclosure, $\gamma$-semiclosed set, $\gamma$ - $b$-closure, $\gamma$-b-closed set, $\gamma$-b-closed function

\section{The results}

We refute the following Theorems stated by Hussain in [1] through six examples.

Theorem 1.1 ([1, Theorem 3.9]). Let $X$ and $Y$ are topological spaces and function $f$ : $X \rightarrow Y$ be a functions. Then the following statements are equivalent:

(1) $f$ is $\gamma$-b-open.

(2) For each set $B$ of $Y$ and for each $\gamma$-open set $A$ in $X$ such that $f^{-1}(B) \subseteq A$, there is a $\gamma$-b-open set $U$ of $Y$ such that $B \subseteq U$ and $f^{-1}(U) \subseteq A$.

The conclusion mentioned in Theorem 1.1 is incorrect. For example, let $X:=\{a, b, c\}$ and let $\tau:=2^{X}$ be discrete topology on $X$. For $b \in X$, define an operation $\gamma: \tau \rightarrow 2^{X}$ by

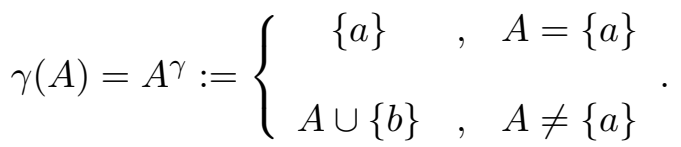

Clearly, $\gamma$-open sets in $X$ are $\emptyset, X,\{a\},\{b\},\{a, b\},\{b, c\}$. Calculations show that $B O_{\gamma}(X)=$ $\{\emptyset, X,\{a\},\{b\},\{a, b\},\{b, c\}\}$. Also, let $Y:=\{1,2,3\}$ and $\sigma:=\{\emptyset, Y,\{3\},\{1,3\}\}$. For $2 \in Y$, define an operation $\gamma: \sigma \rightarrow 2^{Y}$ by

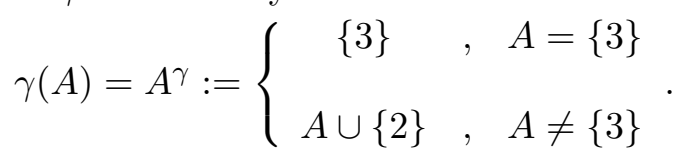

\footnotetext{
*Corresponding Author.

Email addresses: seraperdem123@gmail.com (S. Erdem), murad.ozkoc@mu.edu.tr (M. Özkoç), faicalyacine@gmail.com (F.Y. Issaka)

Received: 05.05.2021; Accepted: 17.09.2021
} 
Clearly, $\gamma$-open sets in $Y$ are $\emptyset, Y,\{3\}$. Calculations show that

$$
B O_{\gamma}(Y)=\{\emptyset, Y,\{3\},\{1,3\},\{2,3\}\} .
$$

Define the function $f: X \rightarrow Y$ by $f:=\{(a, 3),(b, 3),(c, 2)\}$. Simple calculations show that $f$ is $\gamma$-b-open function, but the proposition in $(2)$ is not provided.

Theorem 1.2 ([1, Theorem 2.9]). Let $X$ be a space and $A \subseteq X$. Then

(1) $\operatorname{scl}_{\gamma}(A)=A \cup \operatorname{int}_{\gamma}\left(\operatorname{cl}_{\gamma}(A)\right)$,

(2) $\operatorname{sint}_{\gamma}(A)=A \cap \operatorname{cl}_{\gamma}\left(\operatorname{int}_{\gamma}(A)\right)$.

The conclusion mentioned in Theorem 1.2 is incorrect. For example, let $X:=\{1,2,3\}$ and $\tau:=\{\emptyset, X,\{1\},\{2\},\{1,2\}\}$. Let us consider the operation $\gamma: \tau \rightarrow 2^{X}$ by $\gamma(T):=$ $c l(T)$. Simple calculations show that $\gamma O(X)=\gamma C(X)=S O_{\gamma}(X)=S C_{\gamma}(X)=\{\emptyset, X\}$. For the subset $A:=\{1\} \subseteq X$, we have

$$
\begin{aligned}
\operatorname{scl}_{\gamma}(A) & =\operatorname{scl}_{\gamma}(\{1\}) \\
& =\bigcap\left\{F \mid(\{1\} \subseteq F)\left(F \in S C_{\gamma}(X)\right)\right\} \\
& =\bigcap\{X\} \\
& =X .
\end{aligned}
$$

On the other hand,

$$
\begin{aligned}
A \cup \operatorname{int}_{\gamma}\left(\operatorname{cl}_{\gamma}(A)\right) & =\{1\} \cup \operatorname{int}_{\gamma}\left(\operatorname{cl}_{\gamma}(\{1\})\right) \\
& =\{1\} \cup \operatorname{int}_{\gamma}(\{1,3\}) \\
& =\{1\} \cup\{1\} \\
& =\{1\} .
\end{aligned}
$$

These calculations show that $\operatorname{scl}_{\gamma}(A)=X \neq\{1\}=A \cup \operatorname{int}_{\gamma}\left(\operatorname{cl}_{\gamma}(A)\right)$.

Similarly, for the subset $B:=\{2,3\} \subseteq X$, we have

$$
\begin{aligned}
\operatorname{sint}_{\gamma}(B) & =\operatorname{sint}_{\gamma}(\{2,3\}) \\
& =\bigcup\left\{E \mid(E \subseteq\{2,3\})\left(E \in S O_{\gamma}(X)\right)\right\} \\
& =\bigcup\{\emptyset\} \\
& =\emptyset .
\end{aligned}
$$

On the other hand,

$$
\begin{aligned}
B \cap \operatorname{cl}_{\gamma}\left(\operatorname{int}_{\gamma}(B)\right) & =\{2,3\} \cap \operatorname{cl}_{\gamma}\left(\operatorname{int}_{\gamma}(\{2,3\})\right) \\
& =\{2,3\} \cap \operatorname{cl}_{\gamma}(\{2\}) \\
& =\{2,3\} \cap\{2,3\} \\
& =\{2,3\} .
\end{aligned}
$$

These calculations show that $\operatorname{sint}_{\gamma}(B)=\emptyset \neq\{2,3\}=B \cap \operatorname{cl}_{\gamma}\left(\operatorname{int}_{\gamma}(B)\right)$.

Theorem 1.3 ([1, Theorem 2.10(1)]). Let $X$ be a space and $A \subseteq X$. Then

$$
\operatorname{scl}_{\gamma}\left(\operatorname{sint}_{\gamma}(A)\right)=\operatorname{sint}_{\gamma}(A) \cup \operatorname{int}_{\gamma}\left(\operatorname{cl}_{\gamma}\left(\operatorname{int}_{\gamma}(A)\right)\right) \text {. }
$$

The conclusion mentioned in Theorem 1.3 is incorrect. For example, let $X:=\{1,2,3\}$ and $\tau:=\{\emptyset, X,\{1\},\{2\},\{1,2\}\}$. Let us consider the operation $\gamma: \tau \rightarrow 2^{X}$ by $\gamma(B):=$ 
$\operatorname{cl}(B)$. Simple calculations show that $\gamma O(X)=\gamma C(X)=S O_{\gamma}(X)=S C_{\gamma}(X)=\{\emptyset, X\}$. For the subset $A:=\{2,3\} \subseteq X$, we have

$$
\begin{aligned}
\operatorname{scl}_{\gamma}\left(\operatorname{sint}_{\gamma}(A)\right) & =\operatorname{scl}_{\gamma}\left(\operatorname{sint}_{\gamma}(\{2,3\})\right) \\
& =\operatorname{scl}_{\gamma}\left(\cup\left\{E \mid(E \subseteq\{2,3\})\left(E \in S O_{\gamma}(X)\right)\right\}\right) \\
& =\operatorname{scl}_{\gamma}(\cup\{\emptyset\}) \\
& =\operatorname{scl}_{\gamma}(\emptyset) \\
& =\bigcap\left\{F \mid(\emptyset \subseteq F)\left(F \in S C_{\gamma}(X)\right)\right\} \\
& =\bigcap\{\emptyset, X\} \\
& =\emptyset .
\end{aligned}
$$

On the other hand,

$$
\begin{aligned}
\operatorname{sint}_{\gamma}(A) \cup \operatorname{int}_{\gamma}\left(\operatorname{cl}_{\gamma}\left(\operatorname{int}_{\gamma}(A)\right)\right) & =\operatorname{sint}_{\gamma}(\{2,3\}) \cup \operatorname{int}_{\gamma}\left(\operatorname{cl}_{\gamma}\left(\operatorname{int}_{\gamma}(\{2,3\})\right)\right) \\
& =\emptyset \cup \operatorname{int}_{\gamma}\left(\operatorname{cl}_{\gamma}(\{2\})\right) \\
& =\operatorname{int}_{\gamma}(\{2,3\}) \\
& =\{2\} .
\end{aligned}
$$

These calculations show that $\operatorname{scl}_{\gamma}\left(\operatorname{sint}_{\gamma}(A)\right)=\emptyset \neq\{2\}=\operatorname{sint}_{\gamma}(A) \cup \operatorname{int}_{\gamma}\left(\operatorname{cl}_{\gamma}\left(\operatorname{int}_{\gamma}(A)\right)\right)$.

Theorem 1.4 ([1, Theorem 2.21(9)(10)]). Let $X$ be a space and $A \subseteq X$. Then

(1) $\operatorname{sint}_{\gamma}\left(b c l_{\gamma}(A)\right)=\operatorname{scl}_{\gamma}(A) \cap \operatorname{cl}_{\gamma}\left(\operatorname{int}_{\gamma}(A)\right)$,

(2) $\operatorname{scl}_{\gamma}\left(\operatorname{bint}_{\gamma}(A)\right)=\operatorname{sint}_{\gamma}(A) \cup \operatorname{int}_{\gamma}\left(\operatorname{cl}_{\gamma}(A)\right)$.

The conclusion mentioned in Theorem 1.4 is incorrect. For example, let $X:=\{1,2,3\}$ and $\tau:=\{\emptyset, X,\{1\},\{2\},\{1,2\}\}$. Let us consider the operation $\gamma: \tau \rightarrow 2^{X}$ by $\gamma(T):=$ $c l(T)$. Simple calculations show that $\gamma O(X)=\gamma C(X)=S O_{\gamma}(X)=S C_{\gamma}(X)=\{\emptyset, X\}$ and $B O_{\gamma}(X)=B C_{\gamma}(X)=2^{X}$. For the subset $A:=\{2,3\} \subseteq X$, we have

$$
\begin{aligned}
\operatorname{sint}_{\gamma}\left(\operatorname{bcl}_{\gamma}(A)\right) & =\operatorname{sint}_{\gamma}\left(b_{\gamma}(\{2,3\})\right) \\
& =\operatorname{sint}_{\gamma}\left(\bigcap\left\{F \mid(\{2,3\} \subseteq F)\left(F \in B C_{\gamma}(X)\right)\right\}\right) \\
& =\operatorname{sint}_{\gamma}(\bigcap\{\{2,3\}, X\}) \\
& =\operatorname{sint}_{\gamma}(\{2,3\}) \\
& =\bigcup\left\{E \mid(E \subseteq\{2,3\})\left(E \in S O_{\gamma}(X)\right)\right\} \\
& =\bigcup\{\emptyset\} \\
& =\emptyset .
\end{aligned}
$$

On the other hand,

$$
\begin{aligned}
\operatorname{scl}_{\gamma}(A) \cap \operatorname{cl}_{\gamma}\left(\operatorname{int}_{\gamma}(A)\right) & =\operatorname{scl}_{\gamma}(\{2,3\}) \cap \operatorname{cl}_{\gamma}\left(\operatorname{int}_{\gamma}(\{2,3\})\right) \\
& =\left(\bigcap\left\{F \mid(\{2,3\} \subseteq F)\left(F \in S_{\gamma}(X)\right)\right\}\right) \cap \operatorname{cl}_{\gamma}(\{2\}) \\
& =(\bigcap\{X\}) \cap\{2,3\} \\
& =X \cap\{2,3\} \\
& =\{2,3\} .
\end{aligned}
$$

These calculations show that $\operatorname{sint}_{\gamma}\left(b c l_{\gamma}(A)\right)=\emptyset \neq\{2,3\}=\operatorname{scl}_{\gamma}(A) \cap \operatorname{cl}_{\gamma}\left(\right.$ int $\left._{\gamma}(A)\right)$. 
Similarly, for the subset $B:=\{1\} \subseteq X$, we have

$$
\begin{aligned}
\operatorname{scl}_{\gamma}\left(\operatorname{bint}_{\gamma}(B)\right) & =\operatorname{scl}_{\gamma}\left(\operatorname{bint}_{\gamma}(\{1\})\right) \\
& =\operatorname{scl}_{\gamma}\left(\bigcup\left\{E \mid(E \subseteq\{1\})\left(E \in B O_{\gamma}(X)\right)\right\}\right) \\
& =\operatorname{scl}_{\gamma}(\bigcup\{\emptyset,\{1\}\}) \\
& =\operatorname{scl}_{\gamma}(\{1\}) \\
& =\bigcap\left\{F \mid(\{1\} \subseteq F)\left(F \in S C_{\gamma}(X)\right)\right\} \\
& =\bigcap\{X\} \\
& =X .
\end{aligned}
$$

On the other hand,

$$
\begin{aligned}
\operatorname{sint}_{\gamma}(B) \cap \operatorname{int}_{\gamma}\left(c l_{\gamma}(B)\right) & =\operatorname{sint}_{\gamma}(\{1\}) \cap \operatorname{int}_{\gamma}\left(\operatorname{cl}_{\gamma}(\{1\})\right) \\
& =\left(\bigcup\left\{E \mid(E \subseteq\{1\})\left(E \in S O_{\gamma}(X)\right)\right\}\right) \cap \operatorname{int}_{\gamma}(\{1,3\}) \\
& =(\bigcup\{\emptyset\}) \cap\{1\} \\
& =\emptyset \cap\{1\} \\
& =\emptyset .
\end{aligned}
$$

These calculations show that $\operatorname{scl}_{\gamma}\left(\operatorname{bint}_{\gamma}(B)\right)=X \neq \emptyset=\operatorname{sint}_{\gamma}(B) \cap \operatorname{int}_{\gamma}\left(\operatorname{cl}_{\gamma}(B)\right)$.

\section{References}

[1] S. Hussain, On generalized open sets, Hacet. J. Math. Stat. 47 (6), 1438-1446, 2018. 